\title{
Discovery of optical emission from the supernova remnant G 32.8-0.1 (Kes 78)
}

\author{
P. Boumis ${ }^{1}$, E. M. Xilouris ${ }^{1}$, J. Alikakos ${ }^{1,2}$, P. E. Christopoulou ${ }^{2}$, F. Mavromatakis ${ }^{3}$, \\ A. C. Katsiyannis ${ }^{1}$, and C. D. Goudis ${ }^{1,2}$
}

\author{
1 Institute of Astronomy \& Astrophysics, National Observatory of Athens, I. Metaxa \& V. Paulou, P. Penteli, 15236 Athens, Greece \\ e-mail: [ptb; xilouris; johnal; thk; cgoudis]@astro.noa.gr \\ 2 Astronomical Laboratory, Department of Physics, University of Patras, 26500 Rio-Patras, Greece \\ e-mail: pechris@upatras.physics.gr \\ 3 Technological Education Institute of Crete, Department of Sciences, PO Box 1939, GR-710 04 Heraklion, Crete, Greece \\ e-mail: fotis@stef.teiher.gr
}

Received 4 December 2008 / Accepted 18 March 2009

\section{ABSTRACT}

\begin{abstract}
Deep optical CCD images of the supernova remnant G 32.8-0.1 were obtained where filamentary and diffuse emission was discovered. The images were acquired in the emission lines of $\mathrm{H} \alpha+[\mathrm{N} \mathrm{II}]$ and [S II]. Filamentary and diffuse structures are detected in most areas of the remnant, while no significant [O III] emission is present. The flux-calibrated images suggest that the optical emission originates from shock-heated gas since the $[\mathrm{S} \mathrm{II}] / \mathrm{H} \alpha$ ratio is greater than 1.2. The Spitzer images at $8 \mu \mathrm{m}$ and $24 \mu \mathrm{m}$ show a few filamentary structures to be correlated with the optical filaments, while the radio emission at $1.4 \mathrm{GHz}$ in the same area is found to be very well correlated with the brightest optical filaments. The results from deep long-slit spectra also support the origin of the emission being from shock-heated gas ([S II]/H $\alpha>1.5)$. The absence of [O III] emission indicates slow shock velocities into the interstellar "clouds" $\left(\leq 100 \mathrm{~km} \mathrm{~s}^{-1}\right)$, while the $[\mathrm{S} \mathrm{II}] \lambda \lambda 6716 / 6731$ ratio indicates electron densities up to $\sim 200 \mathrm{~cm}^{-3}$. The $\mathrm{H} \alpha$ emission is measured to lie between 1.8 to $4.6 \times 10^{-17} \mathrm{erg} \mathrm{s}^{-1} \mathrm{~cm}^{-2} \operatorname{arcsec}^{-2}$, while from VGPS HI images the distance to the SNR is estimated to be between 6 to $8.5 \mathrm{kpc}$.
\end{abstract}

Key words. ISM: general - ISM: supernova remnants - ISM: individual objects: 32.8-0.1 (Kes 78)

\section{Introduction}

Supernova remnants (SNRs) are important components of a galaxy since during supernova ejecta they release huge amounts of energy and in subsequent phases heavy elements are mixed into the interstellar medium (ISM). Hence, they play an important role in our understanding of the supernova mechanism, the interstellar medium (ISM) and their interaction. The majority of SNRs have been discovered in radio wavelengths from their nonthermal synchrotron emission and only a few of them have been detected in soft X-rays and in the optical (Green 2006). Optical observations (i.e. Boumis et al. 2005, 2008) offer an important tool for the study of the interaction of the shock wave with dense concentrations of gas found in the ISM.

The Galactic SNR G 32.8-0.1 (Kes78) was discovered in a $408 \mathrm{MHz}$ radio continuum survey by Kesteven (1968), where it appears as a small source $\left(20^{\prime} \times 10^{\prime}\right.$ in diameter $)$. Several radio observations have been perfromed at 30.9, 330, 408, 430, 2700, 5000, $10600 \mathrm{MHz}$ by Kassim (1989), Kassim (1992), Caswell et al. (1975), Dickel \& Denoyer (1975), Velusamu \& Kundu (1974), Caswell et al. (1975) and Beckel \& Kundu (1975) showing its non-thermal emission, while a spectral index between 0.5 and 0.78 was established. Observations of the hydroxyl molecule $(\mathrm{OH})$ at $1720 \mathrm{MHz}$ (Koralesky et al. 1998) showed that the emission results from masers that are shock-excited due to the interaction of the SNR and an adjacent molecular cloud. They found a magnetic field of $1.5 \pm 0.3 \mathrm{mG}$ and determined a kinematic distance to the SNR of $7.1 \mathrm{kpc}$. Allakhverdiyev et al. (1983) and Case \& Bhattacharya (1998) using the radio surface brightness - diameter relationship ( $\Sigma$-D) calculated a distance of
$7.1 \mathrm{kpc}$ and $6.3 \mathrm{kpc}$ respectively. Neutral hydrogen observations at $21 \mathrm{~cm}$ (Gosachinskii \& Khersonskii 1985) resulted in a distance of $9 \mathrm{kpc}$, an age of $1.2 \times 10^{5} \mathrm{yr}$ and an explosion energy of $5.3 \times 10^{50} \mathrm{erg}$ for the SNR. In radio surveys of the surrounding region at $1.4 \mathrm{GHz}$ (Manchester et al. 1985), no pulsar of period $\geq 10$ ms was found to be associated with $\mathrm{G} 32.8-0.1$ up to $\sim 1$ mJy. IRAS observations (Saken et al. 1992) showed a barely resolved IR shell to be coincident with the SNR's radio emission. The Spitzer Space Telescope observed the region of interest as part of a large survey to map a large part of the Galactic plane. In a recent study (Reach et al. 2006), the radio emission of Kes 78 was compared with the infrared emission of this part of the sky and the comparison was described as "confused" since no clear correlation was evident.

In this paper we report the optical detection of $\mathrm{G} 32.8-0.1$ and present flux calibrated images in major optical emission lines. Deep long slit spectroscopy was also performed in a number of selected areas of interest. In Sect. 2, information about the observations and data reduction is given, while the results of the imaging and spectroscopic observations are presented in Sect. 3 and 4. In Sect. 5, we compare our data with observations at other wavelengths, in Sect. 6 we discuss the optical properties of the SNR, and in Sect. 7 we summarize the results of this work.

\section{Observations}

A summary and log of our observations are given in Table 1. In the sections below, we describe these observations in detail. 
Table 1. Imaging and spectral log.

\begin{tabular}{|c|c|c|c|c|c|c|}
\hline \multicolumn{7}{|c|}{ IMAGING } \\
\hline Filter & $\begin{array}{l}\lambda_{c} \\
(\AA)\end{array}$ & $\begin{array}{l}\Delta \lambda \\
(\AA)\end{array}$ & $\begin{array}{l}\text { Total exp. time } \\
\text { (s) }\end{array}$ & $\mathrm{N}^{0}$ of diff. fields & Telescope & \\
\hline $\mathrm{H} \alpha+[\mathrm{N}$ II $] 6548 \& 6584 \AA$ & 6570 & 75 & $4800(2)^{a}$ & 1 & $0.3-\mathrm{m}$ & \\
\hline [O III $] 5007 \AA$ & 5005 & 28 & $9600(4)$ & 1 & $0.3-\mathrm{m}$ & \\
\hline [S II] $6716 \& 6731 \AA$ & 6720 & 18 & $9600(4)$ & 1 & $0.3-\mathrm{m}$ & \\
\hline Cont blue & 5470 & 230 & 180 & 1 & $0.3-\mathrm{m}$ & \\
\hline Cont red & 6096 & 134 & 180 & 1 & $0.3-\mathrm{m}$ & \\
\hline $\mathrm{H} \alpha+[\mathrm{N}$ II $] 6548 \& 6584 \AA$ & 6570 & 75 & 2400 & 6 & $1.3-\mathrm{m}$ & \\
\hline Cont red & 6096 & 134 & 180 & 6 & $1.3-\mathrm{m}$ & \\
\hline \multicolumn{7}{|c|}{ SPECTROSCOPY } \\
\hline \multirow[t]{2}{*}{ Area } & \multicolumn{2}{|c|}{ Slit centers } & Total exp. time & Offset $^{b}$ & Aperture length $^{c}$ & Telescope \\
\hline & $\alpha$ & $\delta$ & $(s)$ & $(\operatorname{arcsec})$ & $(\operatorname{arcsec})$ & \\
\hline South I (SI) & $18^{\mathrm{h}} 51^{\mathrm{m}} 40.6^{\mathrm{s}}$ & $-00^{\circ} 18^{\prime} 04^{\prime \prime}$ & $7800(2)$ & $23.6 \mathrm{~N}$ & 13.0 & $1.3-\mathrm{m}$ \\
\hline South II (SII) & $18^{\mathrm{h}} 51^{\mathrm{m}} 40.6^{\mathrm{s}}$ & $-00^{\circ} 18^{\prime} 04^{\prime \prime}$ & $7800(2)$ & $8.3 \mathrm{~S}$ & 31.9 & $1.3-\mathrm{m}$ \\
\hline East I (EI) & $18^{\mathrm{h}} 51^{\mathrm{m}} 48.6^{\mathrm{s}}$ & $-00^{\circ} 09^{\prime} 44^{\prime \prime}$ & 3900 & $37.8 \mathrm{~S}$ & 20.1 & $1.3-\mathrm{m}$ \\
\hline East II (EII) & $18^{\mathrm{h}} 51^{\mathrm{m}} 48.6^{\mathrm{s}}$ & $-00^{\circ} 09^{\prime} 44^{\prime \prime}$ & 3900 & $64.9 \mathrm{~S}$ & 10.6 & $1.3-\mathrm{m}$ \\
\hline
\end{tabular}

${ }^{a}$ Numbers in parentheses represent the number of individual frames.

${ }^{b}$ Spatial offset from the slit center in arcsec: $N$ (=North), $S$ (=South).

${ }^{c}$ Aperture lengths for each area in arcsec.
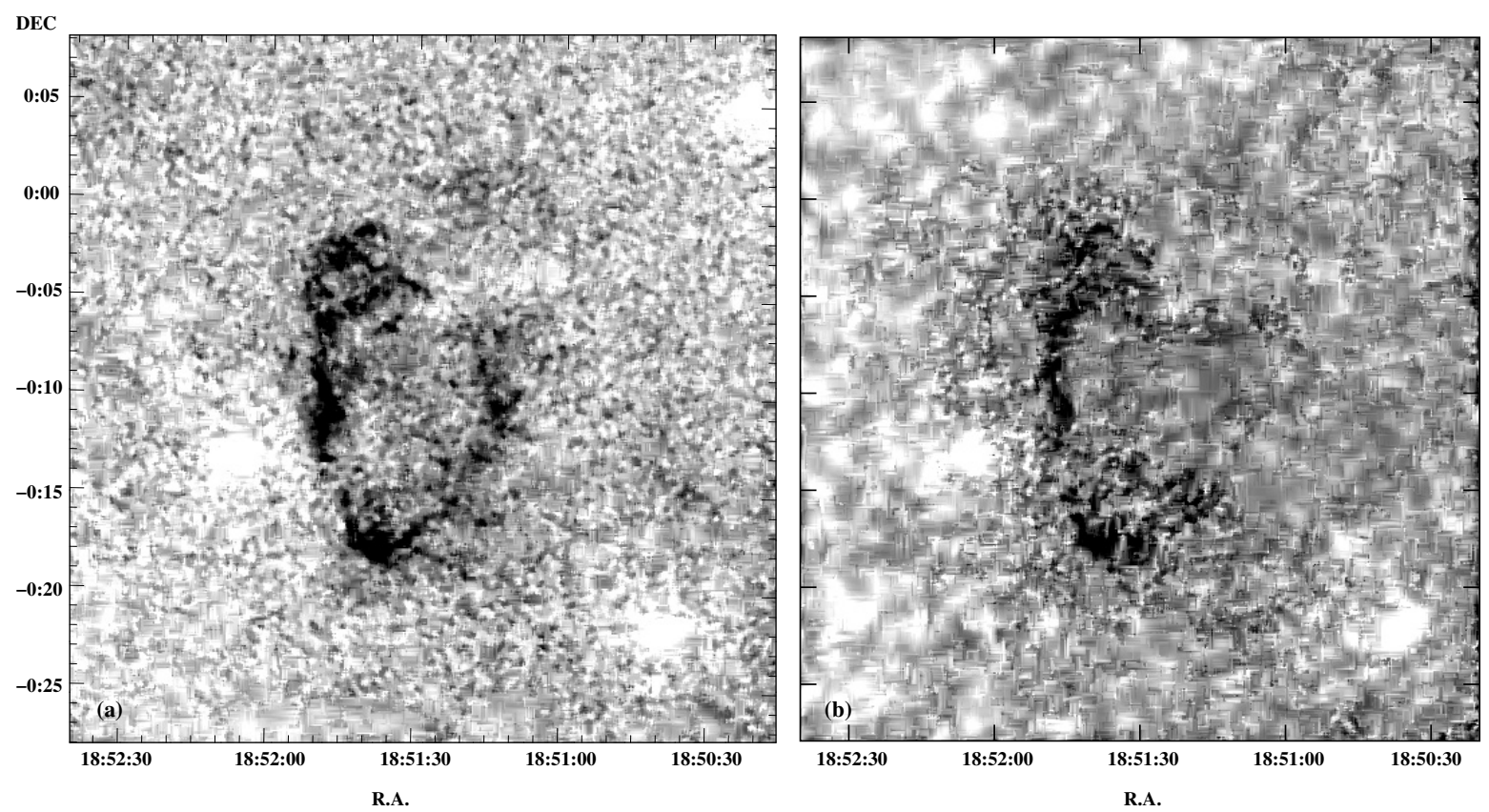

Fig. 1. The G 32.8-0.1 in a) the $\mathrm{H} \alpha+[\mathrm{N} \mathrm{II}]$ and b) the [S II] filter. Shadings run linearly from 0 to $40 \times 10^{-17} \mathrm{erg} \mathrm{s}^{-1} \mathrm{~cm}^{-2}$ arcsec $^{-2}$ and 0 to $25 \times 10^{-17} \mathrm{erg} \mathrm{s}^{-1} \mathrm{~cm}^{-2} \operatorname{arcsec}^{-2}$ in $\left.\mathbf{a}\right)$ and b), respectively. The images have been smoothed to suppress the residuals from the imperfect continuum subtraction.

\subsection{Imaging}

\subsubsection{Wide-field imagery}

The wide-field imagery of $\mathrm{G} 32.8-0.1$ was obtained with the $0.3 \mathrm{~m}$ Schmidt-Cassegrain (f/3.2) telescope at Skinakas Observatory, Crete, Greece on June 7-9, 11 and August 26, 2005. A $1024 \times 1024$ Thomson CCD was used which has a pixel size of $19 \mu \mathrm{m}$ resulting in a $70^{\prime} \times 70^{\prime}$ field of view and an image scale of $4^{\prime \prime}$ per pixel. The area of the remnant was observed with the $\mathrm{H} \alpha+[\mathrm{N} \mathrm{II}]$, [S II], and [O III] filters. The exposure time was set to $2400 \mathrm{~s}$ for each exposure and $180 \mathrm{~s}$ for the continuum red and blue filters. The corresponding continuum images were subtracted from those containing the emission lines to eliminate the confusing star field (see Boumis et al. 2002, for details of this technique). The continuum-subtracted images of the $\mathrm{H} \alpha+[\mathrm{N} \mathrm{II}]$ and $[\mathrm{S} \mathrm{II}]$ emission lines are shown in Figs. 1a and $1 \mathrm{~b}$, respectively.

The IRAF and MIDAS packages were used for the data reduction. All frames were bias-subtracted and flatfield-corrected using a series of well exposed twilight frames. A smoothing of a $5 \times 5$ pixels median box was applied to all images while the stars were removed by appropriately scaling and subtracting the continuum images. The spectrophotometric standards stars HR 5501, HR 7596, HR 7950, HR 8634, and HR9087 (Hamuy et al. 1992) were used for the absolute flux calibration. 
Table 2. Typically measured fluxes over the brightest filaments.

\begin{tabular}{lllll}
\hline \hline & $S$ & $E$ & $N$ & $W$ \\
\hline $\mathrm{H} \alpha+[\mathrm{N}$ II $]$ & 20.8 & 19.8 & 18.7 & 21.3 \\
{$[\mathrm{~S}$ II $]$} & 17.5 & 14.7 & 12.3 & $<6^{a}$ \\
\hline
\end{tabular}

Fluxes in units of $10^{-17} \mathrm{erg} \mathrm{s}^{-1} \mathrm{~cm}^{-2} \operatorname{arcsec}^{-2}$.

Median values over a $40^{\prime \prime} \times 40^{\prime \prime}$ box.

$S=$ (South), $N(=$ North $), W(=$ West $)$ and $E(=$ East $)$.

a $3 \sigma$ upper limit.

The astrometric solution for all data frames was calculated using the Hubble Space Telescope (HST) Guide Star Catalogue (Lasker et al. 1999). All the equatorial coordinates quoted in this work refer to epoch 2000.

\subsubsection{High-resolution imagery}

Optical images at higher angular resolution of G 32.8-0.1 were obtained with the $1.3 \mathrm{~m}$ (f/7.7) Ritchey- Cretien telescope at Skinakas Observatory on July 4-7 and 8-10, 2007, using the $\mathrm{H} \alpha+[\mathrm{N}$ II] interference filter. The detector was a $1024 \times$ 1024 SITe CCD with a field of view of $8.5 \times 8.5 \mathrm{arcmin}^{2}$ and an image scale of $0.5^{\prime \prime}$ per pixel. Six exposures were taken through the $\mathrm{H} \alpha+[\mathrm{N}$ II] filter, each of $2400 \mathrm{~s}$ and six corresponding exposures in the continuum, each of $180 \mathrm{~s}$. After the continuum subtraction, all fields were projected to a common origin on the sky and were subsequently combined to create the final mosaic in $\mathrm{H} \alpha+[\mathrm{N}$ II $]$. Note that the upper right field was observed close to the morning twilight, significantly reducing its signal-to-noise in comparison with the surrounding fields. During the observations, the "seeing" varied between $0.8^{\prime}$ "and 1.5 ", while the full width at half maximum (FWHM) of the star images was between $1.1^{\prime \prime}$ and 2.1". The continuum-subtracted mosaic is shown in Fig. 2.

\subsection{Spectroscopy}

Low dispersion long-slit spectra were obtained with the $1.3 \mathrm{~m}$ telescope at Skinakas Observatory on June 4 and 5 and September 7, 2005. The exposure time was $3900 \mathrm{~s}$. The 1300 line $\mathrm{mm}^{-1}$ grating was used with the $2000 \times 800(13 \mu \mathrm{m})$ SITe CCD covering the range $4750 \AA-6815 \AA$. The $1 \AA$ pixel $^{-1}$ and the dispersion of 1300 line $\mathrm{mm}^{-1}$ result in a spectral resolution of $\sim 8$ and $\sim 11 \AA$ in the red and blue wavelengths, respectively. The slit has a width of 7'.7 and length of 7!9 and in all cases was oriented in the south-north direction. The coordinates of the slit centers of each spectrum are given in Table 1. For the absolute flux calibration the spectrophotometric standard stars HR 4468, HR 5501, HR 7596, HR 7950, HR 8634 and HR 9087 were used. The data reduction was performed using the IRAF package.

\section{The $\mathrm{H} \alpha+[\mathrm{N} \mathrm{II],} \mathrm{[S} \mathrm{II]} \mathrm{and} \mathrm{[O} \mathrm{II]} \mathrm{emission} \mathrm{line}$ images}

Optical emission was detected for the first time from this remnant. The most interesting regions lie in the south, east and north, where bright filamentary and faint diffuse structures are present (between $\alpha \simeq 18^{\mathrm{h}} 51^{\mathrm{m}} 35^{\mathrm{s}}, \delta \simeq-00^{\circ} 17^{\prime} 30^{\prime \prime} ; \alpha \simeq 18^{\mathrm{h}} 51^{\mathrm{m}} 51^{\mathrm{s}}$, $\delta \simeq-00^{\circ} 08^{\prime} 40^{\prime \prime}$ and $\left.\alpha \simeq 18^{\mathrm{h}} 51^{\mathrm{m}} 41^{\mathrm{s}}, \delta \simeq-00^{\circ} 01^{\prime} 20^{\prime \prime}\right)$, which all are very well correlated with the radio emission. The bright $\mathrm{H} \alpha+[\mathrm{N}$ II] filaments cover most of the emission found in radio wavelengths and extend for $\sim 16^{\prime}$ from south to north through the east part of the SNR. In contrast to the above results, diffuse emission was mainly detected in the west with only one bright filament between $\alpha \simeq 18^{\mathrm{h}} 51^{\mathrm{m}} 09^{\mathrm{s}}, \delta \simeq-00^{\circ} 09^{\prime} 45^{\prime \prime}$ and $\alpha \simeq 18^{\mathrm{h}} 51^{\mathrm{m}} 14^{\mathrm{s}}, \delta \simeq-00^{\circ} 10^{\prime} 48^{\prime \prime}$ ( $\sim 1^{\prime}$ long). No significant emission was found in the image of the [O III] medium ionization line and therefore it is not shown here. The morphology of the $[\mathrm{S} \mathrm{II}]$ image is generally similar to, though not as bright as, that of the $\mathrm{H} \alpha+[\mathrm{N}$ II] image. We detected [S II] emission where most of the $\mathrm{H} \alpha+[\mathrm{N} \mathrm{II}]$ emission was found with filamentary bright structures in the south and east areas, while diffuse emission characterizes the rest of the remnant's emission.

The flux calibrated images of $\mathrm{H} \alpha+[\mathrm{N} \mathrm{II}]$ and $[\mathrm{S} \mathrm{II}]$ provide a first indication of the nature of the observed emission (see Table 2). A study of these images shows that all parts of the optical remnant originate from shock heated gas since we estimate ratios $[\mathrm{S} \mathrm{II}] / \mathrm{H} \alpha \geq 1.2$ consistent with the spectral measurements. In particular, the eastern and southern areas show $[\mathrm{S} \mathrm{II}] / \mathrm{H} \alpha \sim 1.5$ and 1.7 respectively, while the northern area shows $[\mathrm{S} \mathrm{II}] / \mathrm{H} \alpha \sim 1.3$. Only the western area does not show strong [S II] emission, hence only upper limits are given.

\section{The optical spectra from $\mathrm{G} 32.8-0.1$}

Deep low-resolution spectra were taken on the brightest optical filaments in the eastern and southern parts of the SNR (their exact position are given in Table 1). In Table 3, we present the relative line fluxes taken from two different apertures along the slit. In particular, apertures I and II have an offset (see Table 1) north or south of the slit center because they are free of field stars and at the same time they include sufficient line emission to allow for an accurate determination of the line fluxes. The background extraction aperture was taken towards the northern or the southern ends of the slit depending on the filament's position within the slit.

The measured line fluxes indicate emission from shockheated gas, since $[\mathrm{S} \mathrm{II}] / \mathrm{H} \alpha>1.5$. Furthermore, the $[\mathrm{N}$ II $] / \mathrm{H} \alpha$ ratio, which takes values between 1.3 and 2.2 (see Table 3), falls well inside the range expected for an SNR (Fesen et al. 1985). The signal-to-noise ratios do not include calibration errors, which are less than 10 percent. Typical spectra from the east (E1) and south (SII) are shown in Fig. 3. The absolute $\mathrm{H} \alpha$ flux covers a range of values from 1.8 to $4.6 \times 10^{-17} \mathrm{erg} \mathrm{s}^{-1} \mathrm{~cm}^{-2} \operatorname{arcsec}^{-2}$. The [S II] $\lambda \lambda$ 6716/6731 ratio that was measured between 1.3 and 1.5 indicates electron densities below $150 \mathrm{~cm}^{-3}$ (Osterbrock \& Ferland 2006). However, taking into account the statistical errors on the sulfur lines, it is found that electron densities up to $200 \mathrm{~cm}^{-3}$ are allowed (Shaw \& Dufour 1995). [O III] emission was not detected, while the very weak $\mathrm{H} \beta$ emission and the absence of the [O III] line suggest significant interstellar attenuation of the optical emission. The absence of [O III] emission can also be explained by slow shocks propagating into the interstellar clouds ( $\leq 100 \mathrm{~km} \mathrm{~s}^{-1}$; Hartigan et al. 1987) since higher velocity shocks should produce detectable [O III] emission.

\section{Observations at other wavelengths}

The optical emission matches the radio emission of G 32.8-0.1 very well at $1.4 \mathrm{GHz}$, suggesting their association (Fig. 4). The observed filaments are located close to the outer edge of the radio contours but the low resolution of the radio images does not 


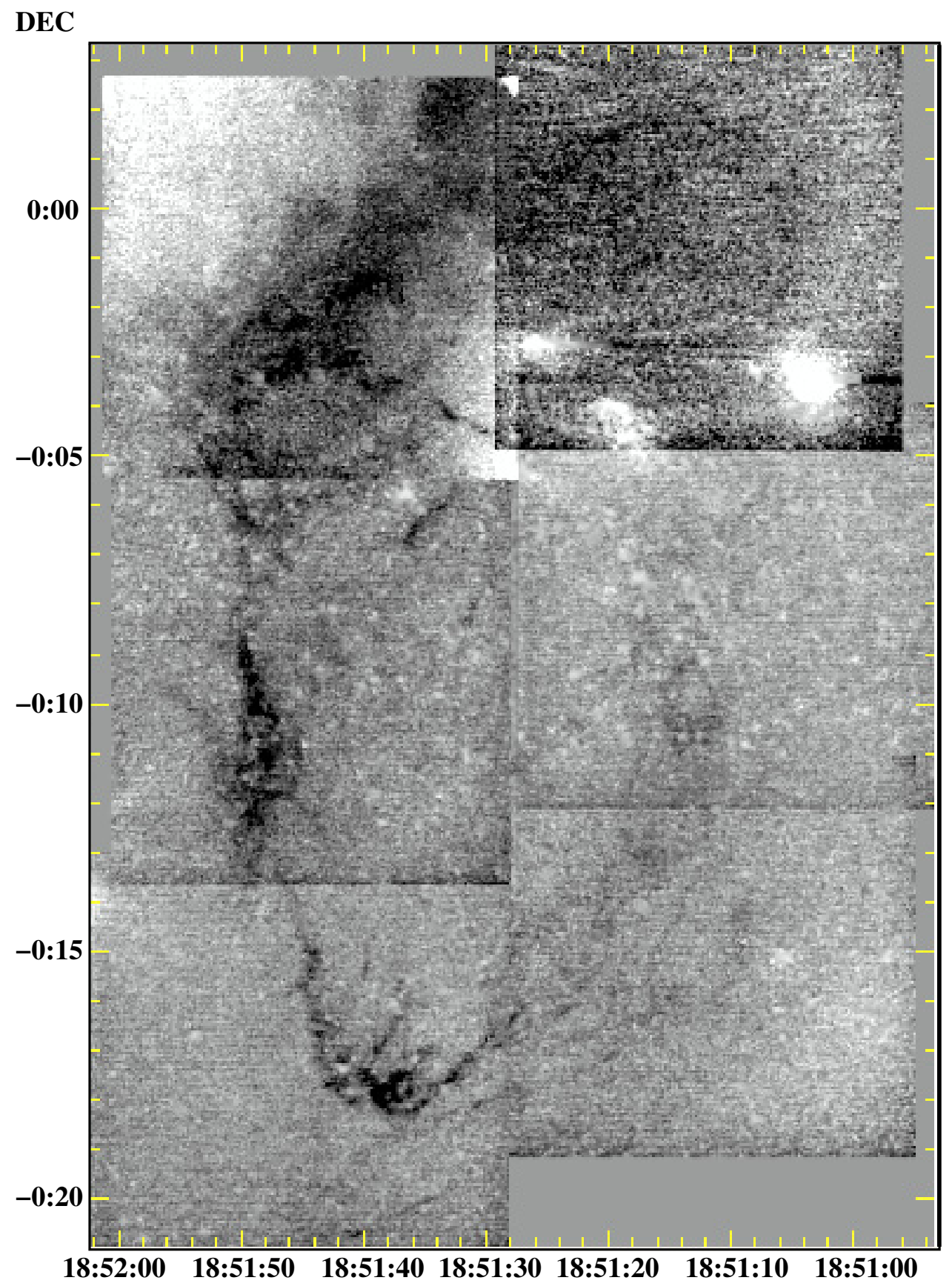

R.A.

Fig. 2. The continuum-subtracted mosaic of G 32.8-0.1 taken with the 1.3-m telescope in $\mathrm{H} \alpha+[\mathrm{N}$ II]. The image has been smoothed to suppress the residuals from the imperfect continuum subtraction.

allow us to determine the relative position of the filament with respect to the shock front.

We investigate possible correlations of the optical emission with infrared emission. The region of interest has been observed with the Spitzer Space Telescope in both $8 \mu \mathrm{m}$ and $24 \mu \mathrm{m}$ bands as part of the large surveys GLIMPSE and MIPSGAL (Benjamin et al. 2003; and Carey et al. 2005, respectively) along the Galactic plane. The superb resolution of Spitzer in these wavelengths allowed us to perform a very careful comparison between optical and infrared emission and look for possible correlations. In Fig. 5, we present a composite image of the $\mathrm{H} \alpha$ optical emission (red), the $24 \mu \mathrm{m}$ emission (green) and the $8 \mu \mathrm{m}$ emission (blue). It is evident that although there is quite a lot of structure and confusion in the background due to neighboring emission regions (as already pointed out by Reach et al. 2006), there are regions (e.g. in the North, North-West and South) where the optical emission overlaps with infrared (mostly with the $8 \mu \mathrm{m}$ ) emission. We also argue that the discontinuity of the shape of the remnant in the North-West part may be due to heavy obscuration due to dust clouds seen in the infrared.

New HI kinematics data are available from the VLA Galactic Plane Survey (VGPS; Stil et al. 2006). The distribution of the atomic gas was examined in detail by searching radial velocities in the range from -113 to $165 \mathrm{~km} \mathrm{~s}^{-1}$ and looking for signs of 
Table 3. Relative line fluxes.

\begin{tabular}{|c|c|c|c|c|c|c|c|c|}
\hline \multirow[b]{2}{*}{ Line $(\AA)$} & \multicolumn{2}{|c|}{ Area SI } & \multicolumn{2}{|c|}{ Area SII } & \multicolumn{2}{|c|}{ Area EI } & \multicolumn{2}{|c|}{ Area EII } \\
\hline & $F^{a}$ & $S / N^{b}$ & $F$ & $S / N$ & $F$ & $S / N$ & $F$ & $S / N$ \\
\hline $\mathrm{H} \beta 4861$ & $<6$ & & $<6$ & & $<4$ & & $<4$ & \\
\hline [O I] 6300 & 43.5 & (23) & 31.3 & (16) & 31.7 & (33) & 67.0 & (18) \\
\hline$[\mathrm{N}$ II $] 6548$ & 50.5 & (25) & 54.5 & (25) & 48.6 & (44) & 29.4 & (9) \\
\hline $\mathrm{H} \alpha 6563$ & 100 & (45) & 100 & (45) & 100 & (83) & 100 & (26) \\
\hline [N II] 6584 & 167.6 & (64) & 167.1 & (67) & 135.0 & (101) & 98.8 & (25) \\
\hline [S II] 6716 & 97.1 & (42) & 93.8 & (41) & 88.1 & (70) & 109.8 & (27) \\
\hline [S II $] 6731$ & 74.8 & (32) & 73.9 & (32) & 66.4 & (58) & 74.4 & (19) \\
\hline Absolute $\mathrm{H} \alpha$ flux ${ }^{c}$ & \multicolumn{2}{|c|}{2.5} & \multicolumn{2}{|c|}{1.9} & \multicolumn{2}{|c|}{4.6} & \multicolumn{2}{|c|}{1.8} \\
\hline$[\mathrm{S} \mathrm{II}] / \mathrm{H} \alpha$ & \multirow{2}{*}{\multicolumn{2}{|c|}{$\begin{array}{l}1.72 \pm 0.05 \\
1.30 \pm 0.05\end{array}$}} & \multicolumn{2}{|c|}{$1.68 \pm 0.05$} & \multicolumn{2}{|c|}{$1.54 \pm 0.03$} & \multicolumn{2}{|c|}{$1.84 \pm 0.09$} \\
\hline$F(6716) / F(6731)$ & & & \multirow{2}{*}{\multicolumn{2}{|c|}{$\begin{array}{l}1.27 \pm 0.05 \\
222+0.06\end{array}$}} & \multirow{2}{*}{\multicolumn{2}{|c|}{$1.33 \pm 0.03$}} & \multicolumn{2}{|c|}{$1.48 \pm 0.09$} \\
\hline$[\mathrm{N}$ II $] / \mathrm{H} \alpha$ & \multicolumn{2}{|c|}{$2.18 \pm 0.06$} & & & & & 1.28 & 0.07 \\
\hline $\mathrm{c}(\mathrm{H} \beta)^{d}$ & \multicolumn{2}{|c|}{$>2.2$} & \multicolumn{2}{|c|}{$>2.2$} & \multicolumn{2}{|c|}{$>2.7$} & \multicolumn{2}{|c|}{$>2.7$} \\
\hline
\end{tabular}

${ }^{a}$ Observed fluxes normalized to $\mathrm{F}(\mathrm{H} \alpha)=100$ and uncorrected for interstellar extinction.

${ }^{b}$ Numbers in parentheses represent the signal to noise ratio of the quoted fluxes.

${ }^{c}$ In units of $10^{-17} \mathrm{erg} \mathrm{s}^{-1} \mathrm{~cm}^{-2} \operatorname{arcsec}^{-2}$.

${ }^{d}$ The logarithmic extinction is derived by $c=1 / 0.348 \times \log \left((\mathrm{H} \alpha / \mathrm{H} \beta)_{\mathrm{obs}} / 2.85\right)$.

Listed fluxes are a signal to noise weighted average of two fluxes for area $S$.

The errors of the emission line ratios are calculated through standard error propagation.

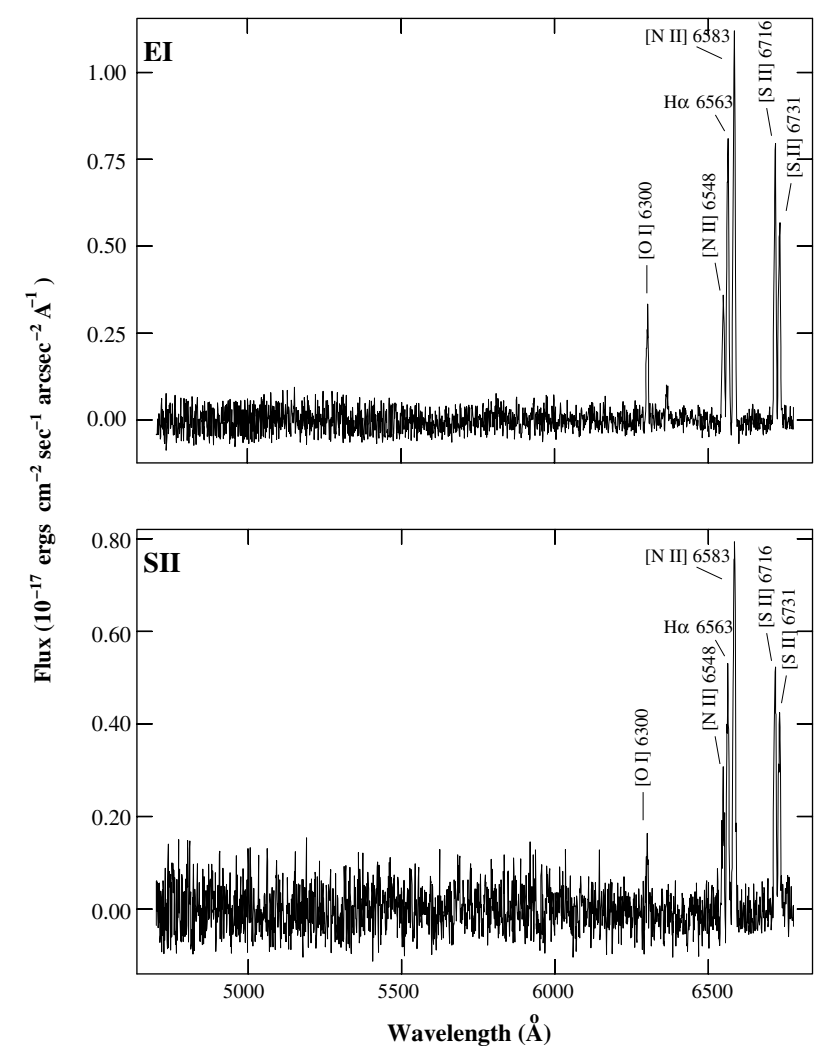

Fig. 3. Long-slit spectra from the east (EI) and south (SII) areas of G 32.8-0.1.

interaction between the expanding shock fronts of the SNR and the surrounding medium. In Fig. 6, we present velocities from six different channels and radio contours on top of the HI emission. The HI images in the range of 10 to $25 \mathrm{~km} \mathrm{~s}^{-1}$ (Figs. 6a-c) are interesting because intense $\mathrm{HI}$ emission is present almost all over the SNR's radio boundaries. If the HI emission is due to the SNR's expanding envelope then using the Galactic rotation curve model and recent measurements of its parameters
$\left(R_{0}=7.6 \mathrm{kpc}\right.$, Eisenhauer et al. 2005; $V_{0}=220 \mathrm{~km} \mathrm{~s}^{-1}$ Feast $\&$ Whitelock 1997) we can estimate distances in the range of 0.8 to $2 \mathrm{kpc}$. At velocities greater than $80 \mathrm{~km} \mathrm{~s}^{-1}$ (Fig. 6d-e) and especially at $113 \mathrm{~km} \mathrm{~s}^{-1}$ (Fig. 6f) features all around the remnant show signs of interaction of the SNR with the HI emission. In that case, the distances are estimated to be in the range of 6 to $8.5 \mathrm{kpc}$.

ROSAT All-sky survey data were also examined, but no significant X-ray emission was detected.

\section{Discussion}

The supernova remnant G 32.8-0.1 appears as an almost complete shell in the radio band; its optical emission marginally correlates with the infrared emission and no X-ray emission has been detected so far. The absence of soft X-ray emission may indicate a low shock temperature and/or a low density of the local interstellar medium. The [O III] flux production depends mainly on the shock velocity and the ionization state of the preshocked gas. Therefore, as mentioned in Sect. 3.2, the absence of [O III] emission may be explained by slow shocks propagating into the ISM. The $\mathrm{H} \alpha+[\mathrm{N}$ II] image best describes the newly detected structures. Sulfur line emission is also detected and generally appears less filamentary and more diffuse than in the $\mathrm{H} \alpha+[\mathrm{N} \mathrm{II}]$ image with their position and shape in agreement with that of the $\mathrm{H} \alpha+[\mathrm{N}$ II]. The absence of [O III] emission does not allow us to determine whether slow shocks travel into ionized gas or whether faster shocks travel into neutral gas (Cox \& Raymond 1985) but we can exclude moderate or fast shocks overtaking ionized gas. The presence of [O I] $6300 \AA$ line emission is also consistent with the emission originating from shock heated gas. Both the calibrated images and the long-slit spectra suggest that the detected emission results from shock heated gas since the $[\mathrm{S} \mathrm{II}] / \mathrm{H} \alpha$ ratio exceeds the empirical SNR criterion value of $0.4-0.5$, while the measured $[\mathrm{N} \mathrm{II}] / \mathrm{H} \alpha$ ratio also confirms this result.

The interstellar extinction c cannot be accurately determined due to the low significance of the $\mathrm{H} \beta$ flux. However, using the $\mathrm{H} \beta$ upper limits (see Table 3), the lower limits on $\mathrm{c}(\mathrm{H} \beta)$ are calculated as 2.2 and 2.7 or $A_{\mathrm{V}}$ of 4.51 and 5.53 for the areas in the South and the East, respectively. Both limits derived from 


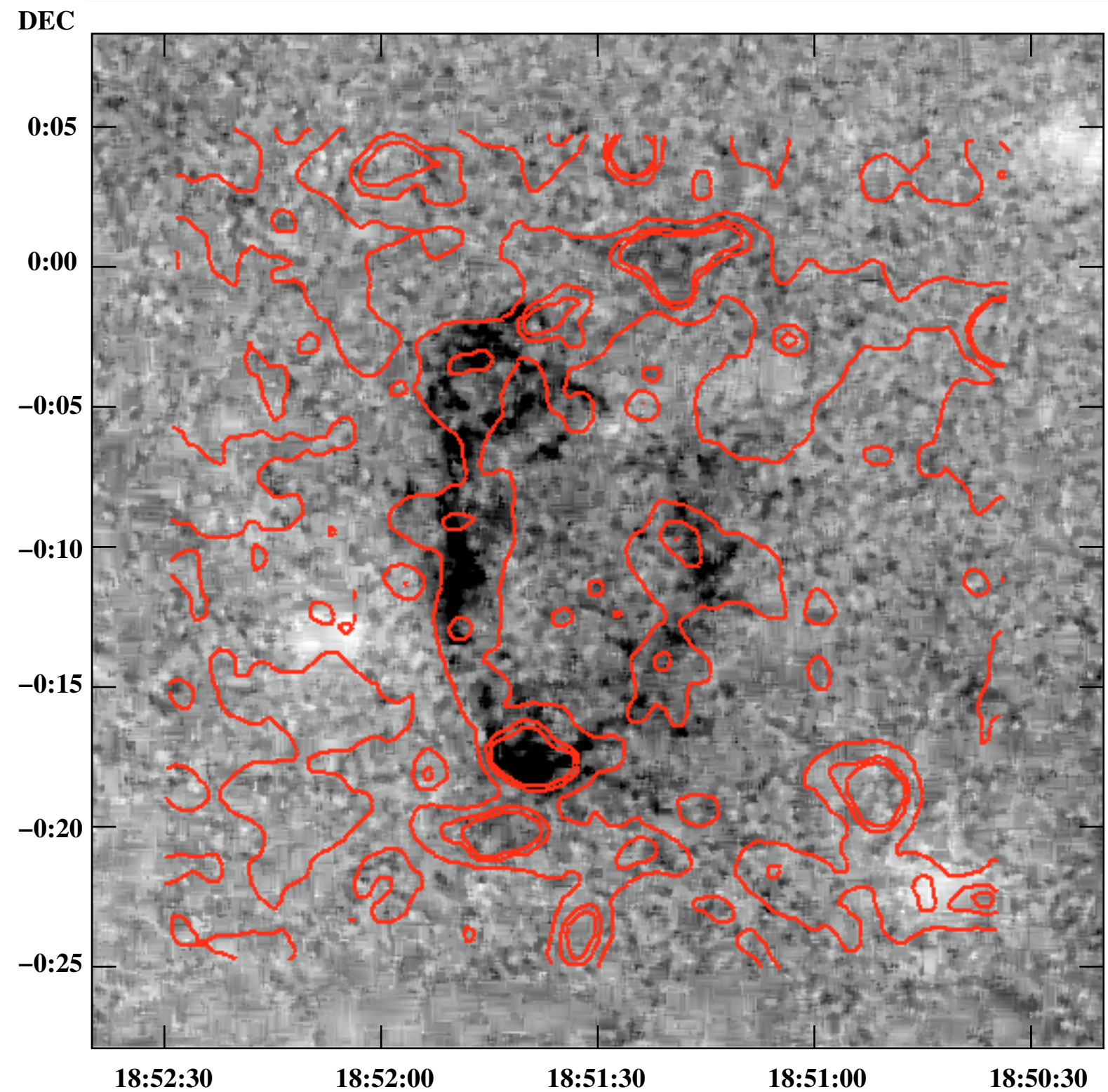

R.A.

Fig. 4. The correlation between the $\mathrm{H} \alpha+[\mathrm{N}$ II] emission, and the radio emission at $1.4 \mathrm{GHz}$ (NVSS - solid red line). The $1.4 \mathrm{GHz}$ radio contours scale linearly from $6 \times 10^{-3} \mathrm{Jy} /$ beam, to $0.02 \mathrm{Jy} /$ beam.

our spectra suggest an area of high interstellar extinction as is expected for an object being at the galactic plane.

Estimated values of $N_{\mathrm{H}} \sim 1.9 \times 10^{22} \mathrm{~cm}^{-2}$ and $N_{\mathrm{H}} \sim$ $1.5 \times 10^{22} \mathrm{~cm}^{-2}$ are given by Dickey \& Lockman (1990) and Kalberla et al. (2005) respectively, for the column density in the direction of G 32.8-0.1. Using the relation of Ryter et al. (1975), we obtain an $N_{\mathrm{H}}>9.9 \times 10^{21} \mathrm{~cm}^{-2}$ and $1.2 \times 10^{22} \mathrm{~cm}^{-2}$ for the c limits calculated from our spectra, respectively. Both values are consistent with the estimated galactic $N_{\mathrm{H}}$ considering the uncertainties involved.

We have also determined the electron density measuring the density-sensitive line ratio of [S II] $\lambda \lambda$ 6716/6731. The measured densities lie below $200 \mathrm{~cm}^{-3}$. Assuming that the temperature is close to $10^{4} \mathrm{~K}$, it is possible to estimate basic SNR parameters. The remnant under investigation has not been studied in detail hence the current stage of its evolution is unknown. Assuming that the remnant is still in the adiabatic phase of its evolution the preshock cloud density $n_{\mathrm{c}}$ can be measured by using the relationship (Dopita 1979)

$n_{[\mathrm{SII}]} \simeq 45 n_{\mathrm{c}} V_{\mathrm{s}}^{2} \mathrm{~cm}^{-3}$

where $n_{[S I I]}$ is the electron density derived from the sulfur line ratio and $V_{\mathrm{s}}$ is the shock velocity into the clouds in units of $100 \mathrm{~km} \mathrm{~s}^{-1}$. Furthermore, the blast wave energy can be expressed in terms of the cloud parameters by using the equation given by McKee \& Cowie (1975)

$E_{51}=2 \times 10^{-5} \beta^{-1} n_{\mathrm{c}} V_{\mathrm{s}}^{2} r_{\mathrm{s}}^{3} \mathrm{erg}$.

The factor $\beta$ is approximately equal to 1 at the blast wave shock, $E_{51}$ is the explosion energy in units of $10^{51} \mathrm{erg}$ and $r_{\mathrm{s}}$ the radius of the remnant in pc. By using the upper limit on the electron density of $200 \mathrm{~cm}^{-3}$, which was derived from our spectra, we 


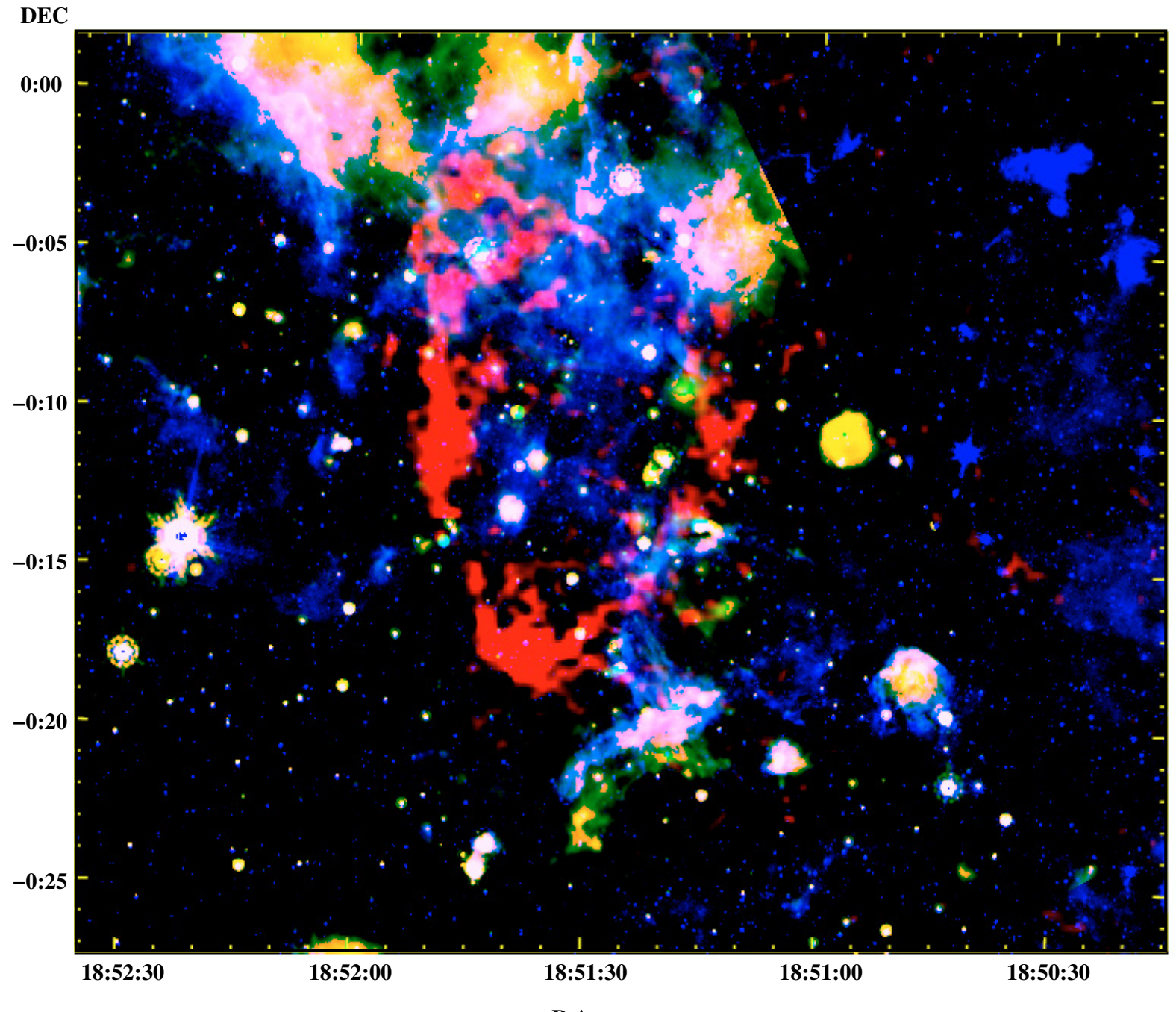

R.A.

Fig. 5. The comparison between the $\mathrm{H} \alpha+[\mathrm{N}$ II] emission (red), and the infrared emission at Spitzer $8 \mu$ (blue) and $24 \mu$ (green). The $24 \mu \mathrm{m}$ emission shadings run logarithmic from 45 to $105 \mathrm{MJy} / \mathrm{sr}$ and the $8 \mu \mathrm{m}$ emission from 66 to $240 \mathrm{MJy} / \mathrm{sr}$.

obtain from Eq. (1) that $n_{\mathrm{c}} V_{\mathrm{s}}^{2}<4.4$. Then Eq. (2) becomes $E_{51}<$ $1.1 \times 10^{-3} D_{1 \mathrm{kpc}}^{3}$, where $D_{1 \mathrm{kpc}}$ the distance to the remnant in units of $1 \mathrm{kpc}$.

Since there are no other measurements of the interstellar density $n_{0}$, values of 0.1 and 1.0 will be examined. Following the result of Eq. (2) and assuming the value of 0.53 (Gosachinskii $\&$ Khersonskii 1985) for the supernova explosion energy $\left(E_{51}\right)$, we find that the remnant may lie at a distance greater than $7.9 \mathrm{kpc}$. Then, the higher interstellar density of $\sim 1 \mathrm{~cm}^{-3}$ suggests that the column density is greater than $7.9 \times 10^{21} \mathrm{~cm}^{-2}$, while for $n_{0} \approx 0.1 \mathrm{~cm}^{-3}$ it becomes greater than $7.9 \times 10^{20} \mathrm{~cm}^{-2}$. Combining the previous results and assuming that the column density is found in the range of $1.5-1.9 \times 10^{22} \mathrm{~cm}^{-2}$, then the higher interstellar density seems to be more probable. The observed HI morphology provides clear evidence of the interaction of the SNR with the surrounding ISM. However, in all cases, the actual distance depends also on the adopted Galactic rotation curve even in the case where a correlation is well established. Therefore, the values found is Sect. 5 may be slightly over (or under) estimated, since some contribution of HI that is not associated with the SNR may be included. The kinematic distances in the second case are in aggrement with previous estimates of the distance to the SNR, based on several methods summarized in
Sect. 1. Thus, a distance to the SNR between 6 to $8.5 \mathrm{kpc}$ might be more plausible. However, more observations are needed (e.g. kinematic) in order to confidently determine the current stage of evolution of G 32.8-0.1.

\section{Conclusions}

Optical emission from the supernova remnant G 32.8-0.1 was detected for the first time with both filamentary and diffuse structure present. The bright optical filaments correlate very well with the remnant's radio emission at $1.4 \mathrm{GHz}$ suggesting their association, while correlation evidences is also shown with the Spitzer Space Telescope mostly at $8 \mu \mathrm{m}$. The flux-calibrated images and the long-slit spectra indicate that the emission arises from shock-heated gas. The surrounding ISM was explored through the HI $21 \mathrm{~cm}$ emission line. From these observation we adopted a distance to the SNR of between 6 to $8.5 \mathrm{kpc}$. Finally, an upper limit for the electron density of $200 \mathrm{~cm}^{-3}$ is calculated.

Acknowledgements. The authors would like to thank John Meaburn and Jeroen Stil for their helpful discussions on $V_{\mathrm{LSR}}$ calculation. J.A. acknowledges funding by the European Union and the Greek Ministry of Development in the framework of the programme "Promotion of Excellence in Research Institutes (2nd Part)". Skinakas Observatory is a collaborative project of the University of Crete, the 


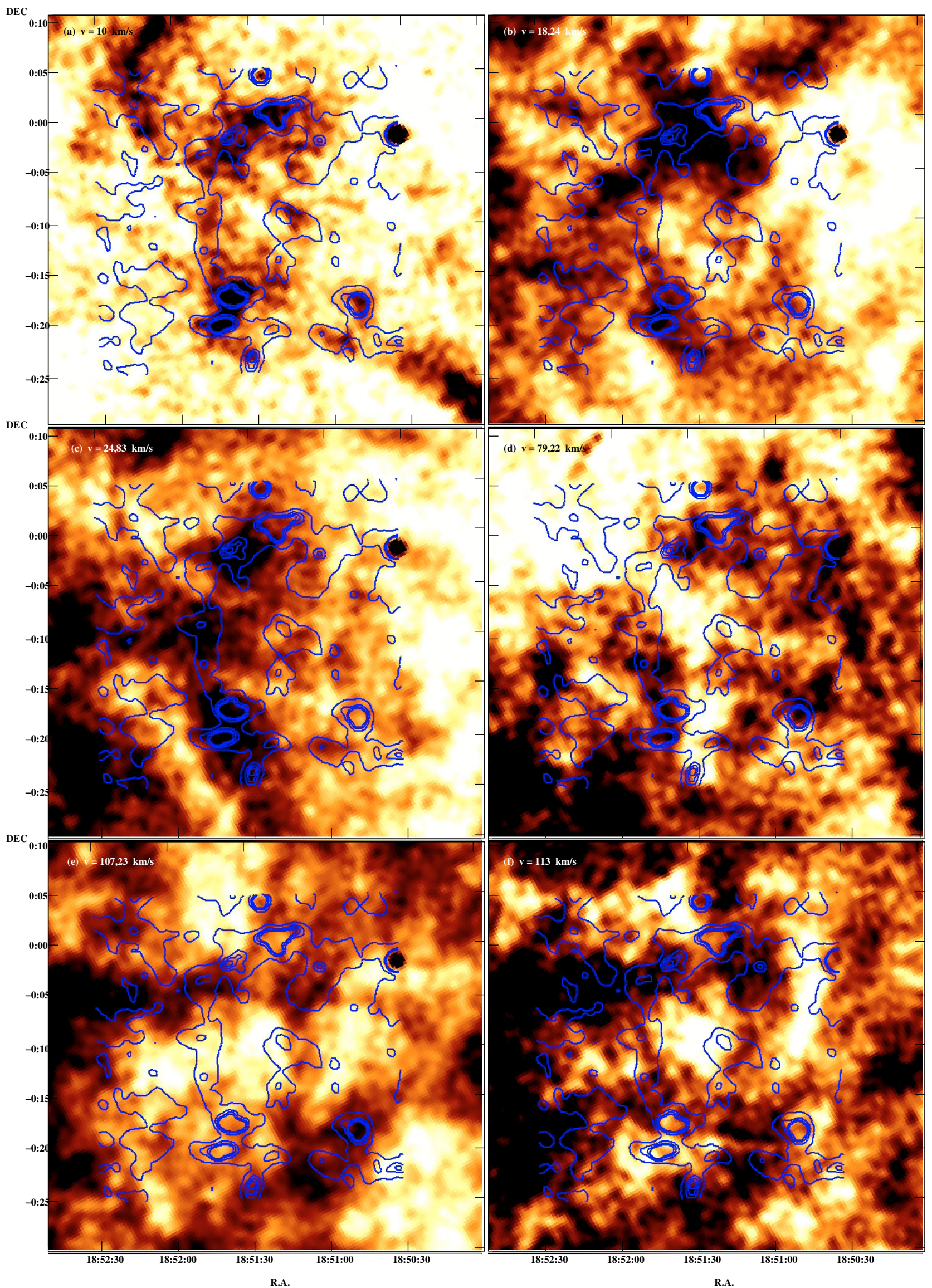

Fig. 6. VGPS images of the $21 \mathrm{~cm}$ line correlated with the radio emission of G 32.8-0.1. The LSR velocities are indicated on the top left of each panel. The VGPS images scale linearly from 10 to $130 \mathrm{~K}$ for panels a)-c) and from 0 to $120 \mathrm{~K}$ for panels d)-f). The $1.4 \mathrm{GHz}$ radio contours (blue line) scale linearly from $6 \times 10^{-3} \mathrm{Jy} /$ beam, to $0.02 \mathrm{Jy} / \mathrm{beam}$. 
Foundation for Research and Technology-Hellas and the Max-Planck-Institut für Extraterrestrische Physik. The National Radio Astronomy Observatory is a facility of the National Science Foundation operated under cooperative agreement by Associated Universities, Inc.

\section{References}

Allakhverdiyev, A. O., Guseinov, O. Kh., Kasumov, F. K., \& Yusifov, I. M. 1983, Ap\&SS, 97, 287

Becker, R. H., \& Kundu, M. R. 1975, AJ, 80, 679

Benjamin, R. A., Churchwell, E., Babler, B. L., et al. 2003, PASP, 115, 953

Boumis, P., Mavromatakis, F., Palaiologou, E. V., \& Becker, W. 2002, A\&A, 396, 225

Boumis, P., Mavromatakis, F., Xilouris, E. M., et al. 2005, A\&A, 443, 175

Boumis, P., Alikakos, J., Christopoulou, P. E., et al. 2008, A\&A, 481, 705

Carey, S. J., et al., 2005, A\&AS, 207, 63.33

Case, G. L., \& Bhattacharya, D. 1998, ApJ, 504, 761

Caswell, J. L., Clark, D. H., \& Crawford, D. F., 1975, AuJPA, 39

Dickel, J. R., \& DeNoyer, L. K. 1975, AJ, 80, 437

Dickey, J. M., \& Lockman, F. J. 1990, ARAA, 28, 215

Dopita, M. A. 1979, ApJS, 40, 455

Eisenhauer, F., Genzel, R., Alexander, T., et al. 2005, ApJ, 628, 246

Feast, M., \& Whitelock, P. 1997, MNRAS, 291, 683

Fesen, R. A., Blair, W. P., \& Kirshner, R. P. 1985, ApJ, 292, 29
Gosachinskij, I. V., \& Khersonskij, V. K. 1985, Ap\&SS, 108, 303

Green, D. A. 2006, A Catalog of Galactic Supernova Remnants (2006 April version), Mullard Radio Astronomy Observatory Cambridge

Hamuy, M., Walker, A. R., Suntzeff, N. B., et al. 1992, PASP, 104, 533

Hartigan, P., Raymond, J., \& Hartmann, L. 1987, ApJ, 316, 323

Kalberla, P. M. W., Burton, W. B., Hartmann, D., et al. 2005, A\&A, 440, 775

Kassim, N. E. 1989, ApJS, 71, 799

Kassim, N. E. 1992, AJ, 103, 943

Kesteven, M. J. L. 1968, AuJPh, 21, 369

Koralesky, B., Frail, D. A., Goss, W. M., Claussen, M. J., \& Green, A. J. 1998, AJ, 116, 1323

Lasker, B. M., Russel, J. N., \& Jenkner, H. 1999, in the HST Guide Star Catalog, version 1.1-ACT, The Association of Universities for Research in Astronomy, Inc

Manchester, R. N., D’Amico, N., \& Tuohy, I. R. 1985, MNRAS, 212, 975

McKee, C. F., \& Cowie, L. L. 1975, ApJ, 195, 715

Olling, R. P., \& Merrifield, M. R., 1998, MNRAS, 297,943

Osterbrock, D. E., \& Ferland, G. J. 2006, Astrophysics of gaseous nebulae and AGN, 2nd edn (Sausalito, CA: University Science Books)

Reach, W. T., Rho, J., Tappe, A., et al., 2006, AJ, 131, 1479

Ryter, C., Cesarsky, C. J., \& Audouze, J. 1975, ApJ, 198, 103

Saken, J, M., Fesen, R. A., \& Shull, J. M. 1992, ApJS, 81, 715

Shaw, R. A., \& Dufour, R. J. 1995, PASP, 107, 896

Stil, J. M., Taylor, A. R., Dickey, J. M., et al. 2006, AJ, 132, 1158

Velusamy, T., \& Kundu, M. R. 1974, A\&A, 32, 375 